\title{
Iterative Refinement of Range Images with Anisotropic Error Distribution
}

\author{
Ryusuke Sagawa $^{\dagger} \quad$ Takeshi Oishi $^{\dagger} \quad$ Atsushi Nakazawa $^{\dagger}$ \\ Ryo Kurazume ${ }^{\ddagger}$ Katsushi Ikeuchi ${ }^{\dagger}$ \\ $\dagger$ The University of Tokyo $\ddagger$ Kyushu University
}

\begin{abstract}
We propose a method which refines the range measurement of range finders by computing correspondences of vertices of multiple range images acquired from various viewpoints. Our method assumes that a range image acquired by a laser range finder has anisotropic error distribution which is parallel to the ray direction. Thus, we find corresponding points of range images along with the ray direction. We iteratively converge range images to minimize the distance of corresponding points. We demonstrate the effectiveness of our method by presenting the experimental results of artificial and real range data. Also we show that our method refines a 3D shape more accurately as opposed to that achieved by using the Gaussian filter.
\end{abstract}

\section{Introduction}

Large scale 3D modeling technology has become popular and is often used for modeling industrial plants, buildings, cultural heritage objects, and so on. Using this technology, enables many project scientists to digitize large scale cultural heritage objects or natural scenes.

The $3 \mathrm{D}$ object modeling is accomplished by performing the following three steps:

(1) Acquiring the range images (Scanning).

(2) Aligning of many range images acquired from different viewpoints (Aligning).

(3) Re-generating the unified meshes (Merging).

Usually, during the scanning process To ensure that all of the $3 \mathrm{D}$ shape data is acquired, several range images are taken of each portion of the object surface.

For the alignment of the small objects, the geometrical relationship between range images can be easily identified because the objects' motions are controlled by using rotation tables or manipulators. However, that relationship cannot be acquired for large objects; in such cases, range image matching techniques are used for the alignment. Many studies have been devoted to achieving this purpose. Besl proposed a feature point-based matching method [1], while Chen's method is based on the evaluation between the point and the polygons. Neugebauer proposed the idea of 'simultaneous registration' that aligns range images simultaneously to avoid the error accumulation of the pairwise alignment methods [6]. A similar idea was proposed by Nishino et al. [7].

A merging procedure produces unified meshes from aligned range images. This is accomplished by concatenating the polygons' borders [10], using deformable surfaces [4] or implicit functions [2, 11]. Wheeler's method uses 'signed distance' to represent the distance from 3D mesh surfaces and their consensus. As the result, the errors and outliers are eliminated.

The errors of the final 3D model come from these factors.

(1) A measurement error on the range images.

(2) A matching error on the alignment process.

(3) The quantization and equalization errors on the merging process.

The type (2) and (3) errors depend on the object shape and the algorithm, and so are solved by selecting a suitable algorithm according to the objects. For the type (1) error, Taubin used the spatial smoothing filter [9], but fine features can be lost during this procedure. Basically, this kind of error cannot be avoided from one range image by using any software algorithms.

Taking many range images of the same surface is the one of the solutions for this problem. Generally, any range measurement system has its characteristic minimum measurement accuracy and error distributions. Wheeler's method is based on this consideration, but is weak for spatially high resolution range images. The signed distance is calculated along the normal direction of the surface. If the normal directions are not responsible because of a measurement error, then the final merging result is also not responsible.

We propose a method to avoid this weakness and improve the accuracy of the final 3D model. This reduces the measurement errors on the distance value in the overlapping areas of the aligned range images. By applying this method before the merging process, a much finer 3D model may be acquired. Unlike the existing spatial filtering method, our method is able to not only smooth the 


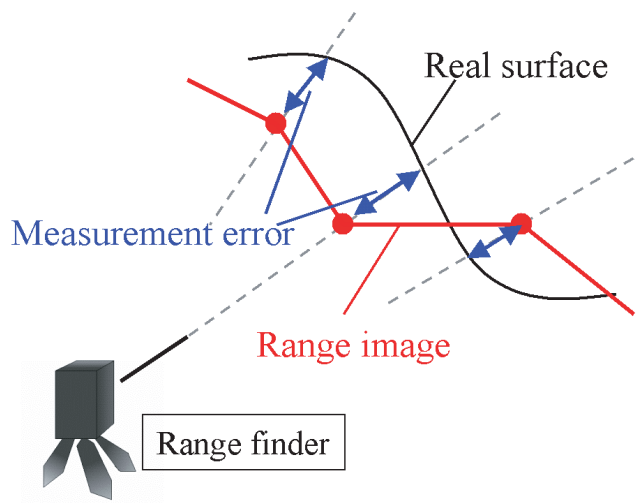

Figure 1: Measurement error

surface of the final 3D mesh model but also to extract fine features. In the following sections, 2 describes our proposed estimation and correction method. 3 shows the result of refinement of artificial and real range data. Finally, we summarize our method in 4 .

\section{Proposed Method}

Our method corrects errors by iterative computation similar to registration techniques like ICP[1]. Let us call the base range image the 'model' and others the 'scenes.' We first search the corresponding points on all scenes of each vertex of the model. Then, we move every vertex of the model respectively to reduce the distance of each correspondence. Our method continues this process until the distances become sufficiently small.

The following pseudo code shows the proposed algorithm:

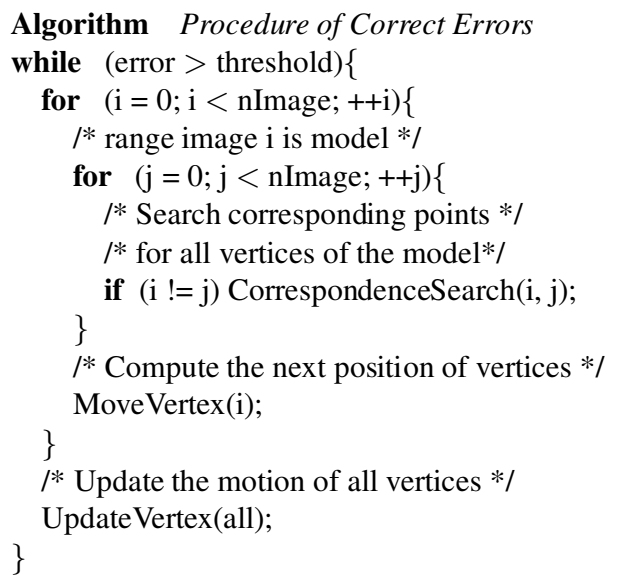

\subsection{Error model of Range Measurement}

Laser range finders measure distance by shooting a laser and receiving its reflection from the target object. The $3 \mathrm{D}$ position of the point of reflection is computed by the

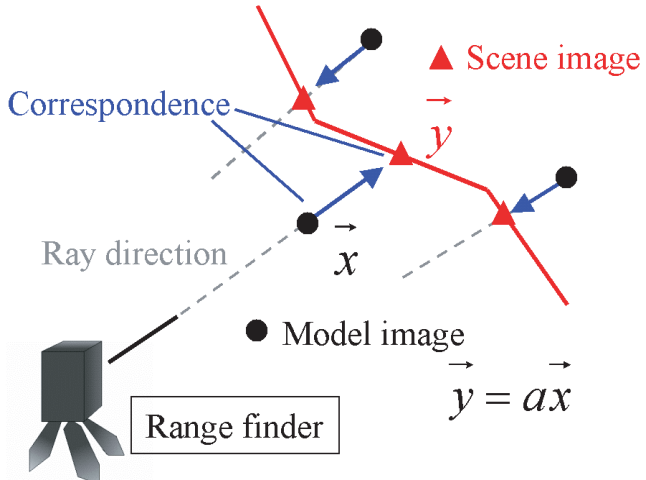

Figure 2: Search correspondence

distance and the ray vector. The error of the $3 \mathrm{D}$ position mainly depends on the error of the distance. The error of the vertical direction to the ray vector, which is caused by the mechanism of the range finder, is much smaller than the error of the distance. Thus, we assume the error of the range measurement by a laser range finder is anisotropic and exists only along the ray vector (Figure 1).

\subsection{Correspondence Search}

Since we assume that error exists only along the ray vector and that range images are completely aligned, our method searches corresponding points along the ray vector. Now, $\vec{x}$ is the vector from the center of the sensor to the vertex of the model and $\vec{y}$ is the vector from the center to the corresponding point of the scene. Then,

$$
\vec{y}=a \vec{x}
$$

where $a$ is the coefficient. Thus, these points are on the same line (Figure 2).

To eliminate wrong correspondences, if the distance of corresponding points is larger than a threshold, we remove the scene point from the correspondence. We use the maximum error of the range finder as the threshold. This correspondence search is computed for every combination of range images.

\subsection{Error Correction}

Errors are corrected by moving each vertex to the new position, which is estimated from the corresponding points. Since the direction of error of each range image is different, some correspondences are not accurate. In the case that the number of overlapped range images is small, it is difficult to estimate the accurate point. Thus, we move each vertex to the weighted average point of the correspondence in order to gradually converge the error. The $k$ th vertex of $i$ th range image $\vec{x}_{i k}$ is moved to the 


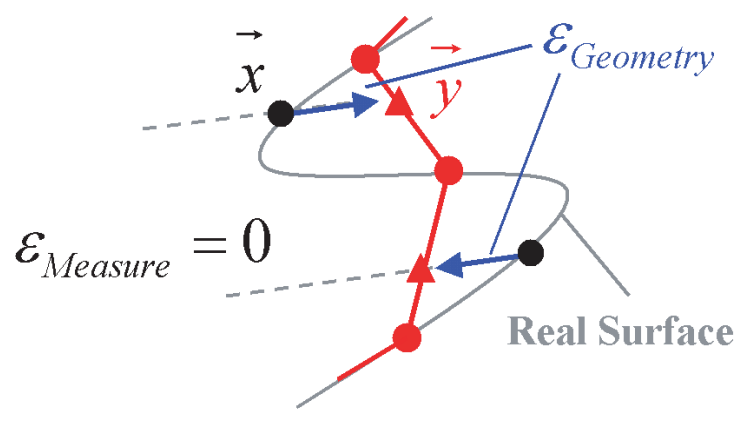

Figure 3: Error by resolution and geometry

weighted average point

$$
\vec{x}_{i k}^{\prime}=(1-w) \cdot \vec{x}_{i k}+w \cdot \frac{1}{n_{i k}-1} \sum_{i \neq j} \vec{y}_{j k}
$$

where $n_{i k}$ is the number of the corresponding points and $w$ is the weight. In this paper, we use $w=0.5$. This process is applied to all vertices of each range image. We reiterate it until the error of correspondence converges sufficiently.

\subsection{Discussion}

The error of corresponding points $\epsilon$ depends on the error of measurement $\epsilon_{\text {Measure }}$ and the error by sparse sampling of a range image $\epsilon_{\text {Geometry }}$.

$$
\epsilon=\epsilon_{\text {Measure }}+\epsilon_{\text {Geometry }}
$$

$\epsilon_{\text {Measure }}$ is corrected by iterative computation. However, $\epsilon_{\text {Geometry }}$ is caused by the curvature of the surface and the sampling interval of a range image.

In Figure 3, the range measurement is noise-free and the vertices of range images are on the real surface (namely $\epsilon_{\text {Measure }}=0$ ); however, the error exists between $\vec{x}$ and $\vec{y}$. Thus, $\epsilon=0$ only if the surface is planar.

$$
\epsilon \begin{cases}=0 & \text { planar area } \\ >0 & \text { otherwise }\end{cases}
$$

Figure 4 shows a 2D example of range images. For simplicity, we assume the new positions of $x_{2}, y_{1}, y_{2}$ after an iteration is computed as

$$
\begin{aligned}
x_{2}^{\prime} & =(1-w) x_{2}+w\left((1-\alpha) y_{1}+\alpha y_{2}\right) \\
y_{1}^{\prime} & =(1-w) y_{1}+w\left((1-\beta) x_{1}+\beta x_{2}\right) \\
y_{2}^{\prime} & =(1-w) y_{2}+w\left((1-\gamma) x_{2}+\gamma x_{3}\right)
\end{aligned}
$$

where $w, \alpha, \beta, \gamma$ are coefficients. Coefficients $\alpha, \beta, \gamma$ are determined by correspondence of $x_{1}, x_{2}, x_{3}, y_{1}$ and $y_{2}$.

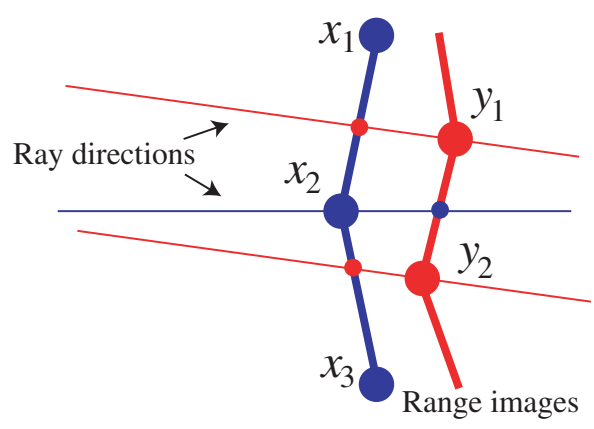

Figure 4: Smoothing effect by iteration

After one more iteration, $x_{2}^{\prime}$ moves to

$$
\begin{aligned}
x_{2}^{\prime \prime}= & (1-w) x_{2}^{\prime}+w\left(\left(1-\alpha^{\prime}\right) y_{1}^{\prime}+\alpha^{\prime} y_{2}^{\prime}\right) \\
= & w^{2}\left(1-\alpha^{\prime}\right)(1-\beta) x_{1}+w^{2} \alpha^{\prime} \gamma x_{3}+ \\
& \left((1-w)^{2}+w^{2}\left(1-\alpha^{\prime}\right) \beta+w^{2} \alpha^{\prime}(1-\gamma)\right) x_{2}+ \\
& w(1-w)\left(2-\alpha-\alpha^{\prime}\right) y_{1}+ \\
& w(1-w)\left(\alpha+\alpha^{\prime}\right) y_{2}
\end{aligned}
$$

where $\alpha^{\prime}$ is a coefficient. Since the equation of $x_{2}^{\prime \prime}$ includes the neighbor vertices $x_{1}, x_{3}$, the smoothing effect occurs during an iteration similar to the smoothing filter. However, the weight of $x_{1}$ and $x_{3}$ in (6) is small compared with that of the smoothing filter, for example,

$$
x_{2}^{\prime}=\alpha x_{1}+\beta x_{2}+(1-\alpha-\beta) x_{3},
$$

which does not include $y_{1}, y_{2}$. Thus, the propagation of the smoothing effect of our method is slower than that of the smoothing filter. In our present implementation, we determine the number of iterations by estimating manually whether the iteration is sufficient.

With regard to the computation cost, most of the computation cost is due to searching for correspondences. Our algorithm searches for correspondences of vertices by rendering all range images from the viewpoint of the reference range image. Thus, the order of computation is $O\left(M N^{2}\right)$ for an iteration of refinement of all range images, where $N$ is the number of range images and $M$ is the number of vertices of a range image.

\section{Experiment}

\subsection{Error Distribution of Laser Range Finder}

Among types of laser range finders, a time-of-flight range finder is useful to measure a distant object with high accuracy. We use a laser range finder of the time-of-flight type, Cyrax 2500 [3] made by Cyra Technologies, Inc. To estimate the error distribution of the Cyrax 2500, we set the range finder in front of a concrete wall and measured 


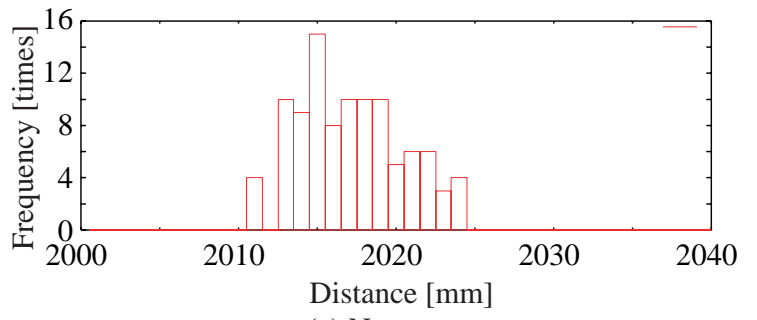

(a) Near range

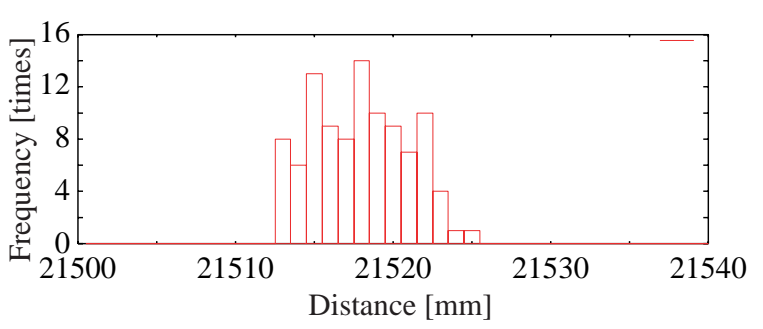

(b) Middle range

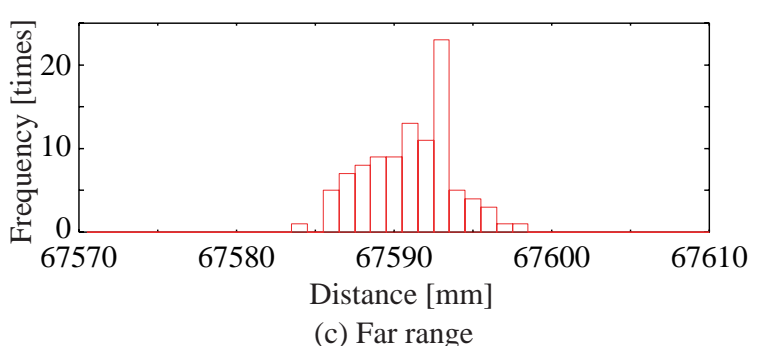

(c) Far range

Figure 5: Distribution of errors of Cyrax 2500

the distance to the wall many times. We tested three configurations of different distances, far range $(67 \mathrm{~m})$, middle range $(20 \mathrm{~m})$ and near range $(2 \mathrm{~m}){ }^{1}$. Figure 5 shows the result of measurement and the average, variance and standard deviation are depicted in Table 1 . The error distribution becomes wide in the near range; however, it can be regarded as a normal distribution with about $3 \mathrm{~mm}$ standard deviation. The maximum error is about $7-8 \mathrm{~mm}$, which is a little larger than the $6 \mathrm{~mm}$ (at $50 \mathrm{~m}$ range) of the catalog specification.

We did not test the error distribution of the vertical direction to the ray vector. According to the catalog, it is $0.25 \mathrm{~mm}$ at $50 \mathrm{~m}$ range $(0.0003$ degree $)$, which is drastically smaller than that of the ray direction. Thus, the error distribution of the range image by Cyrax 2500 depends on the ray direction.

\subsection{Error Correction of Artificial Data}

First, we create artificial range images with random noise and experiment with the error correction. Figure 6(a) shows the model without noise. Its width and height are $40 \mathrm{~cm}$, its depth is $20 \mathrm{~cm}$ and it consists of $100 \times 100$ points. The range image with noise, of which the maxi-

\footnotetext{
${ }^{1}$ The recommended range of Cyrax 2500 is $1.5-50 \mathrm{~m}$.
}

Table 1: Distance measurement error of Cyrax 2500

\begin{tabular}{|l|c|c|}
\hline Average distance $[\mathrm{mm}]$ & Var. $\left[\mathrm{mm}^{2}\right]$ & STD. $[\mathrm{mm}]$ \\
\hline 2017.2 (near) & 11.0 & 3.3 \\
\hline 21518.0 (middle) & 9.1 & 3.0 \\
\hline 67591.1 (far) & 7.7 & 2.8 \\
\hline
\end{tabular}

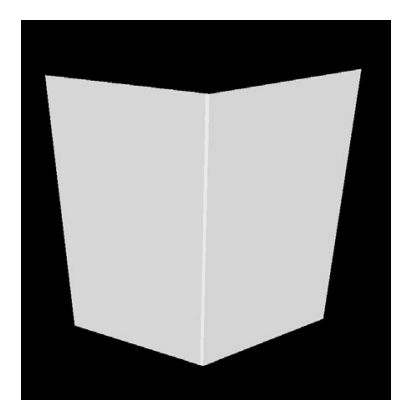

(a)

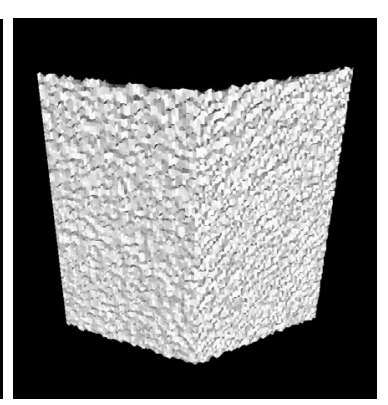

(b)
Figure 6: Artificially created model

mum is $6 \mathrm{~mm}$, is Figure 6(b). We create 10 range images, to which are added noises of different direction. The result of error correction is shown in Figure 7(a). Figure 7(b) is one of range images filtered by Gaussian filter. We can see that our method corrects error sufficiently and preserves edges more accurately than does the Gaussian filter. Figure 8 compares these two results.

We use a PC with a PentiumIII $866 \mathrm{MHz}$ processor and a NVIDIA GeForce3 graphics card. Since our method searches correspondences by rendering range images, it can be accelerated by the use of graphics hardware. The computation time for 20 iterations is 413 seconds.

\subsection{Error Correction of Real Data}

Next, we experiment on the error correction of range images acquired by a laser range finder Cyrax 2400, whose accuracy is the same as that of the Cyrax 2500.

The observed object is the Nara Asuka Great Buddha, which is considered to be the oldest Buddha statue in Japan. Its height is about $2.7 \mathrm{~m}$. Cyrax 2400 is a range finder for long range, and is not suitable for measuring objects of the Asuka Buddha's size. However, because of the bad environment around the Buddha, we cannot use a more accurate range finder for near range. Thus, we measure the Buddha a little apart from it by using the Cyrax 2400 .

We have acquired 9 range images of the front of the Buddha. We align these range images simultaneously by using a robust registration method [7] (see Figure 10). Since the object is relatively small and the range is near, the ob- 


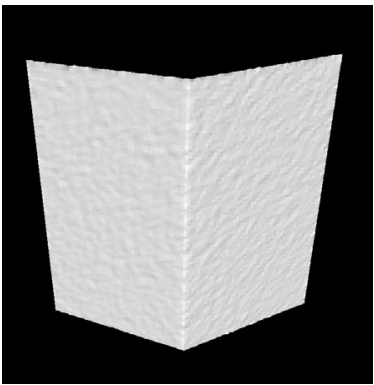

(a)

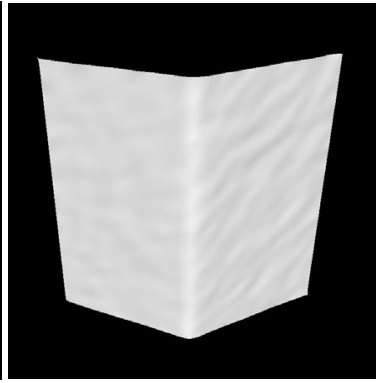

(b)
Figure 7: Refined model

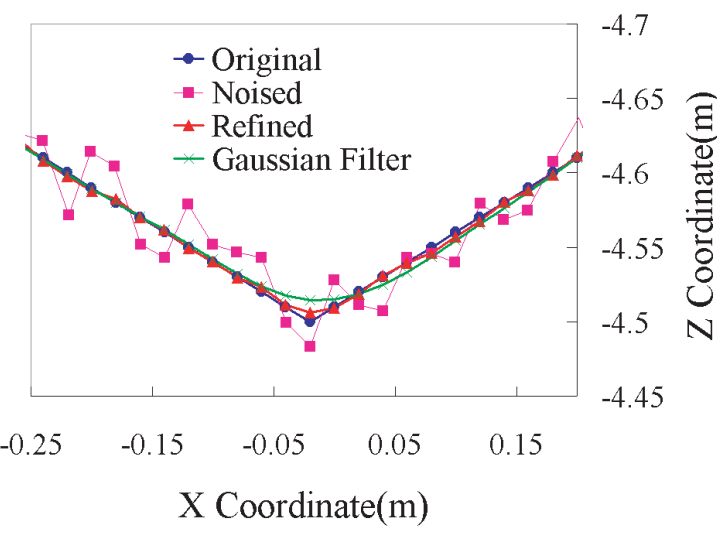

Figure 8: Compare our method and Gaussian filter

vious noise can be seen in the range images.

Figure 11 shows the result of the error correction by our method. The noise has been removed from the model and its surface is smooth. In spite of that, the edges are well preserved because our method is not a smoothing operation such as the Gaussian filter. In Figure 12, the error converges to 0 as the number of iterations increases. The computation time for 20 itrations is 1510 seconds.

Figure 13 shows the results of merging range images using both the original range images and the refined range images. We use the merging technique by Sagawa [8]. Since the method takes consensus of range images using the distance and normal direction [11], it can remove the error caused by measurement and registration. However, in this experiment, it is difficult to correct the error because the range images are too noisy and its normal direction cannot be relied on. We can see that the error remains in the merging result which uses the original range images in Figure 13. On the other hand, the accurate model is reconstructed in the merging result with the

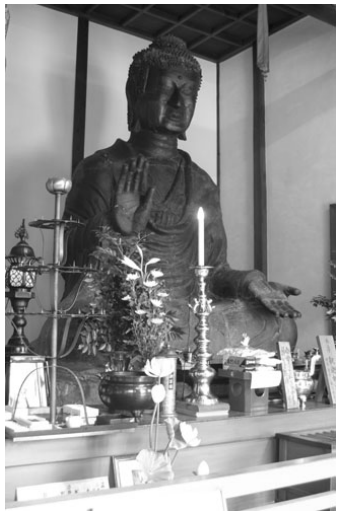

Figure 9: Great Buddha at Asuka temple
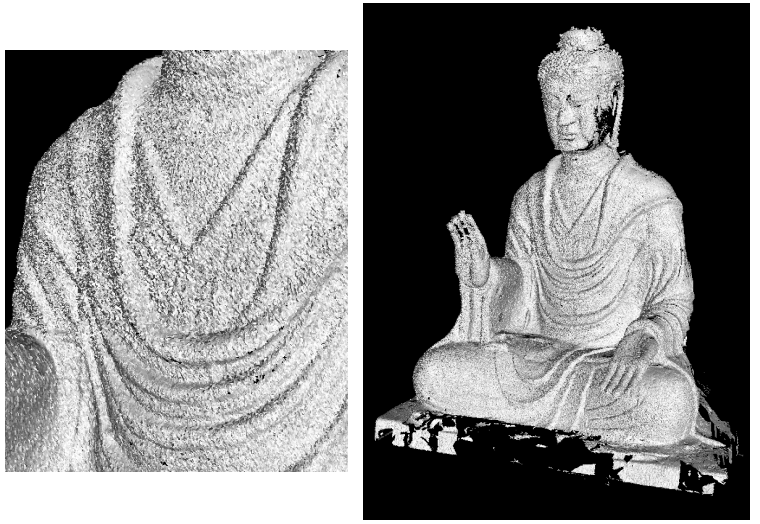

Figure 10: Original range image

refined range images. In the area where range images are not overlapped such as the side of the head, the error is not removed.

We compare our method with the model filtered by the Gaussian filter. Figure 14 shows a part of the model filtered by the Gaussian filter after merging and the model merged from refined range images. The model with the Gaussian filter is smoothed out; however, our method removes noise and preserves the edge of the surface.

Finally, we consider whether our method can be applied to other range finders, for example, a stereo range finding system. We construct a multi-baseline stereo system [5], which consists of 9 cameras. Figure 15 shows one of the camera images and a stereo range image. Since the multibaseline stereo generates a range image for each camera by taking matching images with the other 8 cameras, we generate 9 stereo range images. These range images are pre-aligned by stereo calibration. Thus, our refining process can be applied straightforwardly to the 9 range images. In the raw stereo range image (Figure 16(a)), we can see the step-shaped error caused by quantization of 


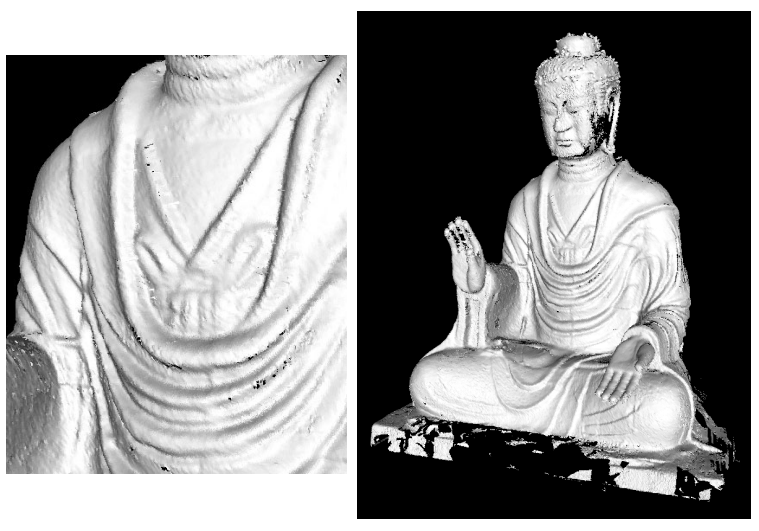

Figure 11: Refined range image

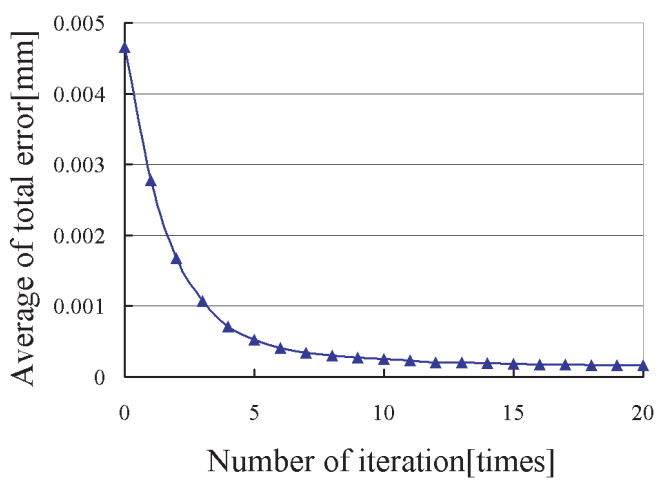

Figure 12: Convergence of error

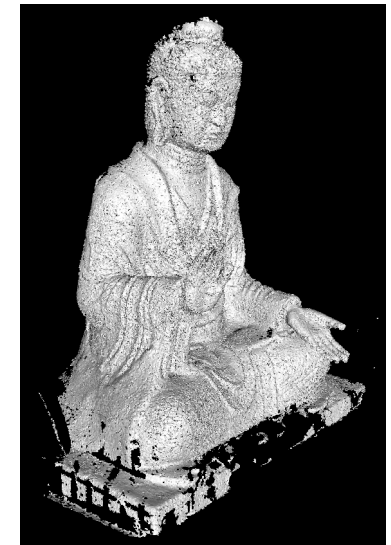

Original

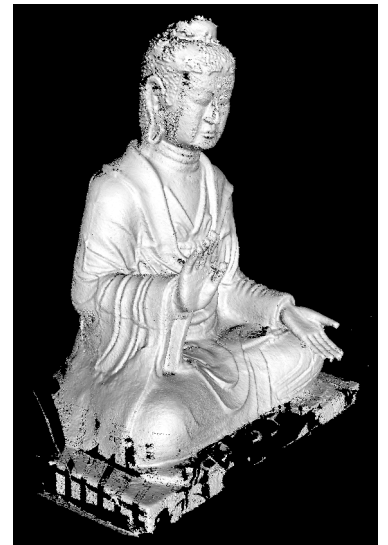

Refined

Figure 13: Results of merging

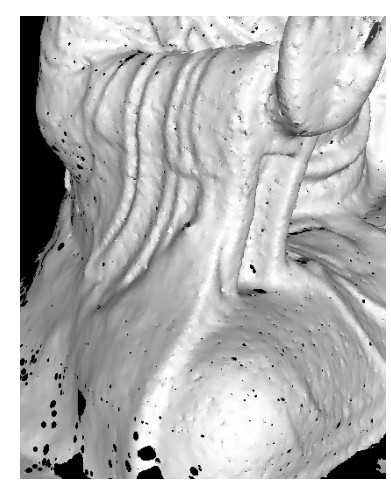

Gaussian Filter

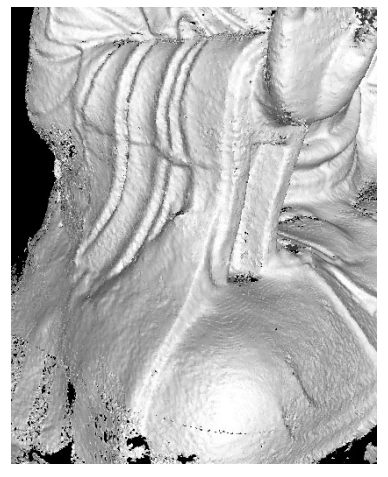

Our Method

Figure 14: Compare with Gaussian filter

images. Figure 16(b) is the refined model after 10 times of the iteration. The step-shaped error is removed after refinement. Also, Figure 16(a) contains a lot of debris due to mismatching. Since the refining process is an estimation of the confidence of range data, we can regard the vertices of the range image which cannot be refined as unreliable vertices. Figure 16(c) is the range image after the unreliable vertices are removed.

\section{Summary}

In this paper, we have proposed an efficient range image refinement method under the consideration of unique error distributions of multiple range images. We described how we applied this method for the modeling of the artificial test object and the actual cultural heritage object from the images acquired by the time-of-flight range sensor. Finally, we applied our method to the range images which are generated by the multi-baseline stereo system. The experimental result shows the validity of this method compared with that of the existing filter-based methods.

\section{References}

[1] P. J. Besl and N. D. McKay. A method for registration of 3-d shapes. IEEE Trans. Pattern Anal. Machine Intell., 14(2):239-256, 1992.

[2] Brian Curless and Marc Levoy. A volumetric method for building complex models from range images. In Proc. SIGGRAPH'96, pages 303-312. ACM, 1996.

[3] http://www.cyra.com.

[4] Martial Hebert, Katsushi Ikeuchi, and Herve Delingette. A spherical representation for recognition of free-form surfaces. IEEE Transactions on Pattern Analysis and Machine Intelligence, 17(7):681-690, 1995.

[5] M.Okutomi and T.Kanade. A multiple-baseline stereo. IEEE Trans. Pattern Analysis and Machine Intelligence, 15(4):353-363, 1993.

[6] P. J. Neugebauer. Geometrical cloning of $3 \mathrm{~d}$ objects via simultaneous registration of multiple range image. In Proc. 


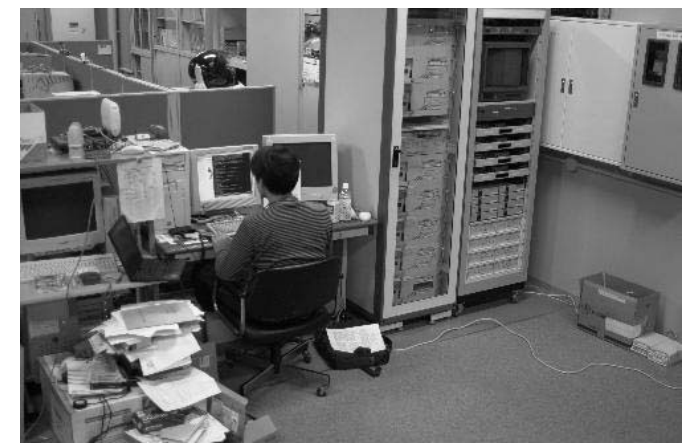

Original camera image

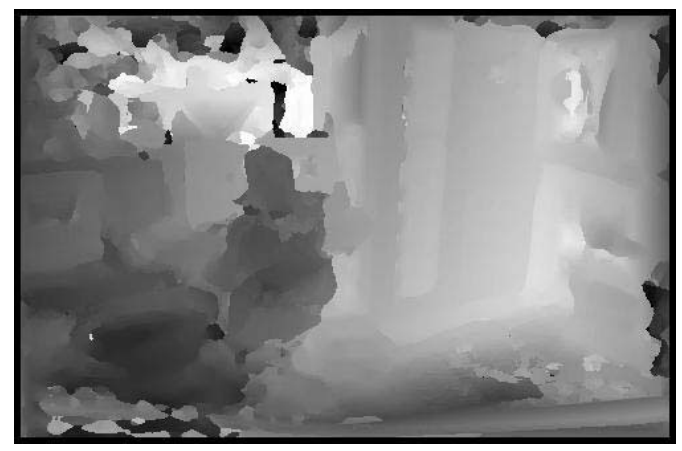

Stereo range image

Figure 15: Range measurement by stereo

of the 1997 Int. Conf. on Shape Modeling and Application (SMA'97), pages 130-139, 1997.

[7] K. Nishino and K. Ikeuchi. Robust simultaneous registration of multiple range images. In Proc. of Fifth Asian Conference on Computer Vision ACCV '02, pages 454-461, 2002.

[8] R. Sagawa, K. Nishino, and K. Ikeuchi. Robust and adaptive integration of multiple range images with photometric attributes. In Proc. IEEE Computer Society Conference on Computer Vision and Pattern Recognition 2001, volume 2, pages 172-179, December 2001.

[9] Gabriel Taubin. A signal processing approach to fair surface design. Computer Graphics, 29(Annual Conference Series):351-358, 1995.

[10] G. Turk and M. Levoy. Zippered polygon meshes from range images. In SIGGRAPH 94, pages 311-318, Jul 1994.

[11] M.D. Wheeler, Y. Sato, and K. Ikeuchi. Consensus surfaces for modeling $3 \mathrm{~d}$ objects from multiple range images. In Proc. International Conference on Computer Vision, January 1998.

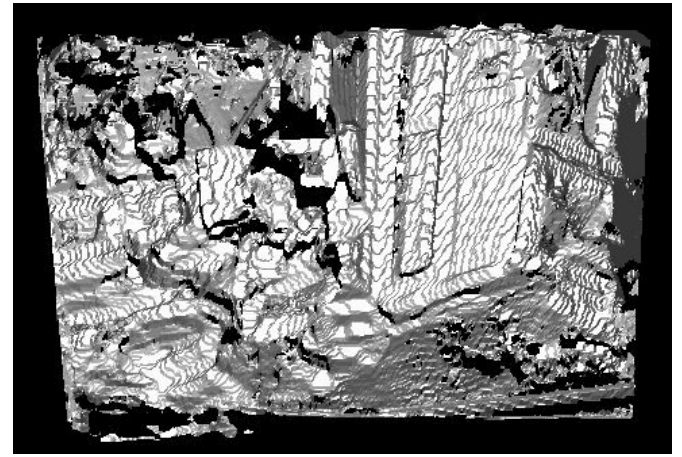

(a)Raw model by stereo

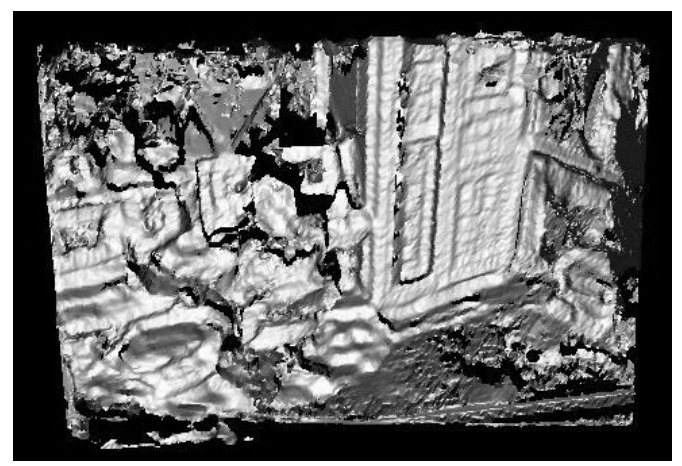

(b)Refined model

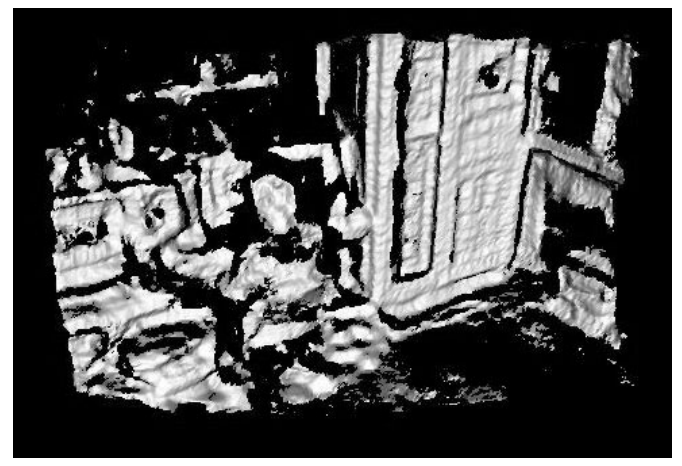

(c)Remove unreliable vertices

Figure 16: Refinement of range image by stereo 